\title{
Analytical exact solutions of heat conduction problems for anisotropic multi-layered media
}

\author{
Chien-Ching $\mathrm{Ma}^{*}$, Shin-Wen Chang \\ Department of Mechanical Engineering, National Taiwan University, Taipei 10617, Taiwan, ROC
}

Received 20 February 2003; received in revised form 31 October 2003

\begin{abstract}
Analytical exact solutions of a fundamental heat conduction problem in anisotropic multi-layered media are presented in this study. The steady-state temperature and heat flux fields in multi-layered media with anisotropic properties in each layer subjected to prescribed temperature on the surfaces are analyzed in detail. Investigations on anisotropic heat conduction problems are tedious due to the presence of many material constants and the complex form of the governing partial differential equation. It is desirable to reduce the dependence on material constants in advance of the analysis of a given boundary value problem. One of the objectives of this study is to develop an effective analytical method to construct full-field solutions in anisotropic multi-layered media. A linear coordinate transformation is introduced to simplify the problem. The linear coordinate transformation reduces the anisotropic multi-layered heat conduction problem to an equivalent isotropic ones without complicating the geometry and boundary conditions of the problem. By using the Fourier transform and the series expansion technique, explicit closed-form solutions of the specific problems are presented in series forms. The numerical results of the temperature and heat flux distributions for anisotropic multi-layered media are provided in full-field configurations.
\end{abstract}

(c) 2003 Elsevier Ltd. All rights reserved.

Keywords: Heat conduction; Anisotropic media; Multi-layered; Coordinate transformation

\section{Introduction}

Many materials in which the thermal conductivity varies with direction are called anisotropic materials. As a result of interesting usage of anisotropic materials in engineering applications, the development of heat conduction in anisotropic media has grown considerably in recent years. To date, few reported results of temperature distribution or heat flux fields in anisotropic media have appeared in the open literature. A number of standard text books (Carslaw and Jaeger [1], Ozisik [2]) have devoted a considerable portion of their contents to heat conduction problems in anisotropic bodies. Most of the earlier works for heat conduction in anisotropic

\footnotetext{
${ }^{*}$ Corresponding author. Tel.: +886-2-2365-9996; fax: +8862-2363-1755.

E-mail address: ccma@ntu.edu.tw (C.-C. Ma).
}

materials have been limited to one-dimensional problems in crystal physics [3,4]. Tauchert and Akoz [5] solved the temperature fields of a two-dimensional anisotropic slab using complex conjugate quantities. Mulholland and Gupta [6] investigated a three-dimensional anisotropic body of arbitrary shape by using coordinate transformations to principal axes. Chang [7] solved the heat conduction problem in a three-dimensional configuration by conventional Fourier transformation. Poon [8] first surveyed the transformation of heat conduction problems in layered composites from anisotropic to orthotropic. Poon et al. [9] extended coordinate transformation of the anisotropic heat conduction problem to isotropic one. Zhang [10] developed a partition-matching technique to solve a two-dimensional anisotropic strip with prescribed temperature on the boundary.

In earlier papers, analytical solutions of anisotropic heat conduction problems have been limited to simple or 


\begin{tabular}{|c|c|c|c|}
\hline \multicolumn{4}{|c|}{ Nomenclature } \\
\hline$a$ & $\begin{array}{l}\text { half region of the prescribed temperature in } \\
\text { the top surface }\end{array}$ & $H_{n}$ & $\begin{array}{l}\text { the thickness of the multi-layered medium } \\
\text { after transformation }\end{array}$ \\
\hline$b$ & $\begin{array}{l}\text { half region of the prescribed temperature in } \\
\text { the bottom surface }\end{array}$ & $\begin{array}{l}k \\
k_{i j}\end{array}$ & $\begin{array}{l}\text { non-dimensional thermal conductivity } \\
\text { thermal conductivity }\end{array}$ \\
\hline$\left(c_{j}, d_{j}\right)$ & undetermined coefficients & $M_{k}^{1}$ & material dependent function on the refrac- \\
\hline$D$ & $\begin{array}{l}\text { the shifted distance for the position of the } \\
\text { concentrated temperature }\end{array}$ & $\left(q_{x}, q_{y}\right)$ & $\begin{array}{l}\text { tion and reflection coefficients } \\
\text { heat flux }\end{array}$ \\
\hline \multicolumn{2}{|c|}{$f(x), g(x)$ arbitrary functions } & $r_{j / j+1}$ & refraction coefficient \\
\hline \multicolumn{2}{|c|}{$F(\omega), G(\omega)$ Fourier transforms of $f(x), g(x)$} & $t_{j / j+1}$ & reflection coefficient \\
\hline$F_{k}^{a}$ & $\begin{array}{l}\text { geometrical dependent function on the } \\
\text { thickness of the layer }\end{array}$ & $\begin{array}{l}T \\
(x, y)\end{array}$ & $\begin{array}{l}\text { temperature } \\
\text { coordinates }\end{array}$ \\
\hline $\boldsymbol{G}_{j / j+1}$ & relative matrix for the coefficients of the & $(X, Y)$ & coordinates after transformation \\
\hline$h_{j}$ & $\begin{array}{l}\text { adjacent layer } \\
\text { vertical distance of the interface for the } j \text { th }\end{array}$ & \multicolumn{2}{|c|}{ Greek symbols } \\
\hline$n_{j}$ & $\begin{array}{l}\text { layer from the top surface } \\
\text { lor the }\end{array}$ & $\alpha, \beta$ & coordinate transform coefficients \\
\hline$H_{j}$ & $\begin{array}{l}\text { vertical distance of the interface for the } j \text { th } \\
\text { layer from the top surface after the coordi- } \\
\text { nate transformation }\end{array}$ & $\omega$ & Fourier transform parameter \\
\hline
\end{tabular}

special cases [2]. In conventional studies of a multidimensional anisotropic medium subjected to distributed temperature or heat flux in or on the media, the analytical solution was obtained by Fourier transformation. It is unlikely to find in most cases the general solutions with respect to each of the spatial variables to satisfy partial differential equations of anisotropic heat conduction equations and boundary conditions. The work of Yan et al. [11] studied two-layered isotropic bodies with homogeneous form of the conduction equation and the Green function solution is used to incorporate the effects of the internal heat source and non-homogeneous boundary conditions. They obtained the series solutions for three-dimensional temperature distribution by Fourier transformation, Laplace transformation and eigenvalue methods. Consequently, it is more difficult to get general analytic solutions satisfying all the boundary conditions for multi-layered anisotropic heat conduction problem because of the continuity of temperature and heat flux on the interface and the cross-derivatives in the governing equation. Therefore, the cross term, which is the crux in solving the anisotropic heat conduction problem, is very troublesome to analyze when one uses conventional solution techniques to solve isotropic heat conduction problems. Due to the mathematical difficulties of the problem, only few solutions for heat conduction in anisotropic media have appeared in the literature and much more work remains to be done.

Exact solutions for heat conduction problems in multi-layered media is of interest in electronic systems and composite materials in a wide variety of modern engineering applications. Consequently, the thermal problems of heat dissipation from devices and systems have become extremely important. The inherent anisotropic nature of layered composites make the analysis more involved than that of isotropic counterpart. However, it may be pointed out that the exact and complete solution for multi-layered bodies of even isotropic media has not been reported to date because of the mathematical difficulties. The mathematical difficulties for heat conduction problem in multi-layered media are caused by the complex form of the governing partial differential equation and by the boundary and continuity conditions associated with the problem. Hsieh and Ma [12] used a linear coordinate transformation to solve the heat conduction problem for a thin-layer medium with anisotropic properties. Exact closed-form solutions of temperature and heat flux fields were obtained by them.

In this study, a two-dimensional heat conduction problem for anisotropic multi-layered media subjected to prescribed temperature on the surfaces is investigated. The number of the layer is arbitrary, the thermal conductivities and the thickness are different in each layer. One of the objectives of this study is to develop an effective methodology to construct the analytical fullfield solution for this problem. Investigations on anisotropic heat conduction problems are tedious due to the presence of many material constants and the crossderivative term of the governing equation. It is desirable to reduce the dependence on material constants in advance of the analysis of a given boundary value problem. A special linear coordinate transformation is 
introduced in this study to simplify the governing heat conduction equation without complicating the continuity and boundary conditions of the problem. Based on this transformation, the original anisotropic multilayered problem is converted to an equivalent isotropic problem with a similar geometrical configuration. Explicit closed-form solutions for the temperature and heat flux are expressed in a series form. Numerical results of the full-field distribution for temperature and heat flux are presented in graphic form and are discussed in detail.

\section{Basic formulation and linear coordinate transformation}

Consider an anisotropic material that is homogeneous and has constant thermo-physical properties. The governing partial differential equation for the heat conduction problem in a two-dimensional Cartesian coordinate system is given by

$k_{11} \frac{\partial^{2} T}{\partial x^{2}}+2 k_{12} \frac{\partial^{2} T}{\partial x \partial y}+k_{22} \frac{\partial^{2} T}{\partial y^{2}}=0$,

where $k_{11}, k_{12}$ and $k_{22}$ are thermal conductivity coefficients, and $T$ is the temperature field. The corresponding heat fluxes are given as

$q_{x}=-k_{11} \frac{\partial T}{\partial x}-k_{12} \frac{\partial T}{\partial y}$,
$q_{y}=-k_{12} \frac{\partial T}{\partial x}-k_{22} \frac{\partial T}{\partial y}$.

Based on irreversible thermo-dynamics, it can be shown that $k_{11} k_{22}>k_{12}^{2}$ and the coefficients $k_{11}$ and $k_{22}$ are positive. The governing equation expressed in Eq. (1) is a general homogeneous second order partial differential equation with constant coefficients. Such a linear partial differential equation can be transformed into the Laplace equation by a linear coordinate transformation. A special linear coordinate transformation is introduced as

$\left[\begin{array}{c}X \\ Y\end{array}\right]=\left[\begin{array}{ll}1 & \alpha \\ 0 & \beta\end{array}\right]\left[\begin{array}{l}x \\ y\end{array}\right]$,

where $\alpha=-\frac{k_{12}}{k_{22}}, \beta=\frac{k}{k_{22}}$ and $k=\sqrt{k_{11} k_{22}-k_{12}^{2}}$. After the coordinate transformation, Eq. (1) can be rewritten as the standard Laplace equation in the $(X, Y)$ coordinate system

$k\left(\frac{\partial^{2} T}{\partial X^{2}}+\frac{\partial^{2} T}{\partial Y^{2}}\right)=0$.

It is interesting to note that the mixed derivative is eliminated from Eq. (1). The relationships between the heat flux in the two coordinate systems are given by

$$
\begin{aligned}
& q_{y}=-k \frac{\partial T}{\partial Y}=q_{Y}, \\
& q_{x}=\alpha k \frac{\partial T}{\partial Y}-\beta k \frac{\partial T}{\partial X}=\beta q_{X}-\alpha q_{Y},
\end{aligned} \quad \text { or } \quad \begin{aligned}
& q_{Y}=-k \frac{\partial T}{\partial Y}, \\
& q_{X}=-k \frac{\partial T}{\partial X} .
\end{aligned}
$$

In a mathematical sense, Eqs. (1) and (2) are transformed to Eqs. (4) and (5) by the linear coordinate transformation expressed in Eq. (3), or in a physical sense, the governing equation (1) and the heat flux and temperature relation (2) of an anisotropic heat conduction problem are converted into an equivalent isotropic problem by properly changing the geometry of the body using the linear coordinate transformation, Eq. (3). The coordinate transformation in Eq. (3) has the following characteristics: (a) it is linear and continuous, (b) an anisotropic problem is converted to an isotropic problem after the transformation, (c) there is no stretch and rotation in the horizontal direction. These important features offer advantages in dealing with straight boundaries and interfaces in the multi-layered system discussed in the present study.

The linear coordinate transformation described by Eq. (3) can be used to solve the anisotropic heat conduction problem for only a single material. However, for a multi-layered anisotropic medium with straight interfaces as shown in Fig. 1, a modification of the linear coordinate transformation will be introduced in the following section to transform the multi-layered anisotropic problem to an equivalent multi-layered isotropic problem.
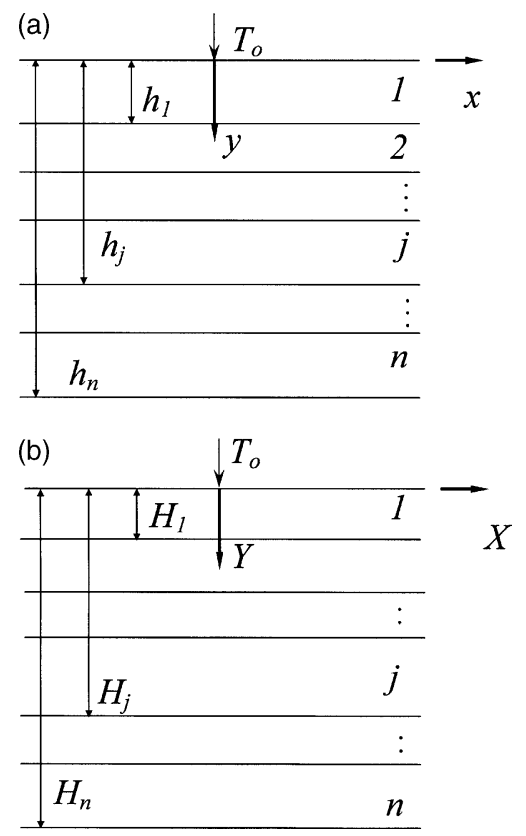

Fig. 1. Configuration and coordinates system of an anisotropic multi-layered medium (a) and after the linear coordinate transformation (b). 


\section{Full-field solutions for anisotropic multi-layered media subjected to concentrated temperature}

In this section, the full-field solutions for the heat conduction problem of an anisotropic $n$-layered medium subjected to a concentrated temperature $T_{0}$ applied on the top surface, as depicted in Fig. 1(a), will be analyzed. The number of the layer is arbitrary, the thermal conductivities and thickness in each layer are different. The steady-state heat conduction equation in each layer is expressed as

$k_{11}^{(j)} \frac{\partial^{2} T^{(j)}}{\partial x^{2}}+2 k_{12}^{(j)} \frac{\partial^{2} T^{(j)}}{\partial x \partial y}+k_{22}^{(j)} \frac{\partial^{2} T^{(j)}}{\partial y^{2}}=0$,

$j=1,2, \ldots, n$.

The boundary conditions on the top and bottom surfaces of the layered medium are

$\left.T^{(1)}\right|_{y=0}=T_{0} \delta(x),\left.\quad T^{(n)}\right|_{y=h_{n}}=0$,

where $\delta()$ is the delta function. The perfect thermal contact condition is assumed for the adjacent layer. The temperature and heat flux continuity conditions at the interface between the $j$ th and $j+1$ th layer yield

$$
\begin{aligned}
& \left.T^{(j)}\right|_{y=h_{j}}=\left.T^{(j+1)}\right|_{y=h_{j}}, \quad j=1,2, \ldots, n-1 . \\
& \left.q_{y}^{(j)}\right|_{y=h_{j}}=\left.q_{y}^{(j+1)}\right|_{y=h_{j}}, \quad
\end{aligned}
$$

In order to maintain the geometry of the layered configuration, the linear coordinate transformation described in Eq. (3) is modified for each layer as follows:

$$
\begin{aligned}
& {\left[\begin{array}{l}
X \\
Y
\end{array}\right]=\left[\begin{array}{ll}
1 & \alpha_{j} \\
0 & \beta_{j}
\end{array}\right]\left[\begin{array}{l}
x \\
y
\end{array}\right]+\sum_{k=1}^{j-1} h_{k}\left[\begin{array}{cc}
\alpha_{k} & -\alpha_{k+1} \\
\beta_{k} & -\beta_{k+1}
\end{array}\right],} \\
& \quad j=1,2, \ldots, n,
\end{aligned}
$$

where $\quad \alpha_{j}=-\frac{k_{12}^{(j)}}{k_{22}^{(j)}}, \quad \beta_{j}=\frac{k_{j}}{k_{22}^{(j)}} \quad$ and $\quad k_{j}=\sqrt{k_{11}^{(j)} k_{22}^{(j)}-k_{12}^{(j)^{2}}}$. Comparing with Eq. (3), the first term in the right-hand side of Eq. (9) retains exactly the same form while the second term with a summation becomes the modified term. The new coordinate transformation possesses the following characteristics: (a) no gaps or overlaps are generated along the interface, (b) no sliding and mismatches occur along the interface. The geometric configuration in the transformed $(X, Y)$ coordinate is shown in Fig. 1(b). Note that while the thickness of each layer is changed, the interfaces are parallel to the $x$-axis. The new geometric configuration after the coordinate transformation is similar to the original problem.

The heat conduction equations in the transformed coordinate for each layer are governed by the standard Laplace equation

$k_{j}\left(\frac{\partial^{2} T^{(j)}}{\partial X^{2}}+\frac{\partial^{2} T^{(j)}}{\partial Y^{2}}\right)=0$.
Furthermore, the temperature $T$ and the heat flux $q_{Y}$ are still continuous along the interfaces in the transformed coordinates,

$\left.T^{(j)}\right|_{Y=H_{j}}=\left.T^{(j+1)}\right|_{Y=H_{j}}, \quad j=1,2, \ldots, n-1$,
$\left.q_{Y}^{(j)}\right|_{Y=H_{j}}=\left.q_{Y}^{(j+1)}\right|_{Y=H_{j}}, \quad$

where $H_{j}=\beta_{j} h_{j}+\sum_{k=1}^{j-1}\left(\beta_{k}-\beta_{k+1}\right) h_{k}$. The top and bottom boundary conditions are expressed as

$\left.T^{(1)}\right|_{Y=0}=T_{0} \delta(X),\left.\quad T^{(n)}\right|_{Y=H_{n}}=0$.

The relations between heat flux field and temperature field expressed in the $(X, Y)$ coordinates within each layer become

$q_{X}^{(j)}(X, Y)=-k_{j} \frac{\partial T^{(j)}(X, Y)}{\partial X}$ $j=1,2, \ldots, n-1$.

$q_{Y}^{(j)}(X, Y)=-k_{j} \frac{\partial T^{(j)}(X, Y)}{\partial Y}$,

The boundary value problem described by Eqs. (10)(13) is similar to the multi-layered problem for an isotropic material. Hence, the linear coordinate transformation presented in Eq. (9) changes the original complicated anisotropic multi-layered problem to the corresponding isotropic multi-layered problem with a similar geometric configuration and boundary conditions.

The boundary value problem will be solved by the Fourier transform technique. Take the Fourier transform pairs defined as

$$
\begin{aligned}
& \widetilde{T}(\omega, Y)=\int_{-\infty}^{\infty} T(X, Y) \mathrm{e}^{-\mathrm{i} \omega X} \mathrm{~d} X \\
& T(X, Y)=\frac{1}{2 \pi} \int_{-\infty}^{\infty} \widetilde{T}(\omega, Y) \mathrm{e}^{\mathrm{i} \omega X} \mathrm{~d} \omega
\end{aligned}
$$

where an overtilde denotes the transformed quantity, $\omega$ is the transform variable, and $\mathrm{i}=\sqrt{-1}$. By applying the Fourier transformation to the governing partial differential equation (10), the equation in transformed domain will be an ordinary differential equation of order two as follows:

$\frac{\mathrm{d}^{2} \widetilde{T}^{(j)}(\omega, Y)}{\mathrm{d} Y^{2}}-\omega^{2} \widetilde{T}^{(j)}(\omega, Y)=0$.

The general solutions of the temperature and heat flux can be presented in the matrix form as

$$
\left[\begin{array}{l}
\widetilde{T}^{(j)} \\
\tilde{q}_{Y}^{(j)}
\end{array}\right]=\left[\begin{array}{cc}
\mathrm{e}^{\omega Y} & \mathrm{e}^{-\omega Y} \\
-k_{j} \omega \mathrm{e}^{\omega Y} & k_{j} \omega \mathrm{e}^{-\omega Y}
\end{array}\right]\left[\begin{array}{l}
c_{j} \\
d_{j}
\end{array}\right] .
$$

Here $c_{j}$ and $d_{j}$ are undetermined coefficients for each layer and can be obtained from the proper boundary and continuity conditions. It is noted that the variable $\omega$ in the above equation is regarded as a parameter.

By using the continuity conditions at the interfaces, the relation for the coefficients of the adjacent layer can be expressed as 
$\left[\begin{array}{l}c_{j} \\ d_{j}\end{array}\right]=\frac{1}{r_{j / j+1}} \boldsymbol{G}_{\mathbf{j} / \mathbf{j}+\mathbf{1}}\left[\begin{array}{c}c_{j+1} \\ d_{j+1}\end{array}\right], \quad j=1,2, \ldots, n-1$,

where

$r_{j / j+1}=\frac{2 k_{j}}{k_{j}+k_{j+1}}$,

$\boldsymbol{G}_{\mathrm{j} / \mathbf{j}+\mathbf{1}}=\left[\begin{array}{cc}1 & t_{j / j+1} \mathrm{e}^{-2 \omega H_{j}} \\ t_{j / j+1} \mathrm{e}^{2 \omega H_{j}} & 1\end{array}\right], \quad t_{j / j+1}=\frac{k_{j}-k_{j+1}}{k_{j}+k_{j+1}}$.

Here $r_{j / j+1}$ and $t_{j / j+1}$ are called the refraction and the reflection coefficients, respectively. Therefore, the relation between the coefficients of the $j$ th layer and the $n$th layer can be expressed as

$\left[\begin{array}{c}c_{j} \\ d_{j}\end{array}\right]=\left[\prod_{k=j}^{n-1}\left(\frac{1}{r_{k / k+1}} \boldsymbol{G}_{\mathbf{k} / \mathbf{k}+\mathbf{1}}\right)\right]\left[\begin{array}{c}c_{n} \\ d_{n}\end{array}\right]$,

where

$\prod_{k=1}^{n} a_{k}=a_{1} \cdot a_{2} \ldots a_{n}$.

By setting $j=1$ in Eq. (18) and applying the boundary conditions as indicated in Eq. (12), the coefficients $c_{n}$ and $d_{n}$ in the $n$th layer are obtained explicitly as follows:

$\left[\begin{array}{l}c_{n} \\ d_{n}\end{array}\right]=\frac{T_{0}}{A_{1}+A_{2}}\left[\begin{array}{c}-\mathrm{e}^{-2 \omega H_{n}} \\ 1\end{array}\right]$,

where $A_{1}$ and $A_{2}$ are expressed in a matrix form as

$\left[\begin{array}{l}A_{1} \\ A_{2}\end{array}\right]=\left[\prod_{k=1}^{n-1}\left(\frac{1}{r_{k / k+1}} \boldsymbol{G}_{\mathbf{k} / \mathbf{k}+\mathbf{1}}\right)\right]\left[\begin{array}{c}-\mathrm{e}^{-2 \omega H_{n}} \\ 1\end{array}\right]$.

The undetermined constants $c_{j}$ and $d_{j}$ for each layer are determined with the aid of the recurrence relations given in Eqs. (18) and (19). After substituting the coefficients $c_{j}$ and $d_{j}$ into Eq. (16), the full-field solutions for each layer are completely determined in the transformed domain. The solutions of temperature and heat flux in the transformed domain for each layer are finally expressed as

$$
\begin{aligned}
{\left[\begin{array}{c}
\widetilde{T}^{(j)} \\
\tilde{q}_{Y}^{(j)}
\end{array}\right]=} & \frac{T_{0}}{A_{1}+A_{2}}\left[\begin{array}{cc}
\mathrm{e}^{\omega Y} & \mathrm{e}^{-\omega Y} \\
-k_{j} \omega \mathrm{e}^{\omega Y} & k_{j} \omega \mathrm{e}^{-\omega Y}
\end{array}\right] \\
& \times\left[\prod_{k=j}^{n-1}\left(\frac{1}{r_{k / k+1}} \boldsymbol{G}_{\mathbf{k} / \mathbf{k}+\mathbf{1}}\right)\right]\left[\begin{array}{c}
-\mathrm{e}^{-2 \omega H_{n}} \\
1
\end{array}\right] .
\end{aligned}
$$

Since the solutions in Fourier transformed domain have been constructed, to inverse the solutions will be the next step. Because of the denominators in Eq. (21), it is impossible to inverse the Fourier transform directly. By examining the structure of the denominator of Eq.
(21), both the numerator and denominator are multiplied by a constant $S=\prod_{k=1}^{n-1} r_{k / k+1}$. Then it becomes,

$$
\begin{aligned}
{\left[\begin{array}{c}
\widetilde{T}^{(j)} \\
\tilde{q}_{Y}^{(j)}
\end{array}\right]=} & \frac{S T_{0}}{S\left(A_{1}+A_{2}\right)}\left[\begin{array}{cc}
\mathrm{e}^{\omega Y} & \mathrm{e}^{-\omega Y} \\
-k_{j} \omega \mathrm{e}^{\omega Y} & k_{j} \omega \mathrm{e}^{-\omega Y}
\end{array}\right] \\
& \times\left[\prod_{k=j}^{n-1}\left(\frac{1}{r_{k / k+1}} \boldsymbol{G}_{\mathbf{k} / \mathbf{k}+\mathbf{1}}\right)\right]\left[\begin{array}{c}
-\mathrm{e}^{-2 \omega H_{n}} \\
1
\end{array}\right] .
\end{aligned}
$$

The denominator in Eq. (22), $S\left(A_{1}+A_{2}\right)$, can be decomposed into the form of $(1-p)$ where $p=1-S\left(A_{1}+A_{2}\right)$. It can be shown that $p<1$ for $\omega>0$. By a series expansion, we obtain $\frac{1}{1-p}=\sum_{l=0}^{\infty} p^{l}$ so that Eq. (22) can be rewritten as

$$
\begin{aligned}
{\left[\begin{array}{c}
\widetilde{T}^{(j)} \\
\tilde{q}_{Y}^{(j)}
\end{array}\right]=} & S T_{0}\left[\begin{array}{cc}
\mathrm{e}^{\omega Y} & \mathrm{e}^{-\omega Y} \\
-k_{j} \omega \mathrm{e}^{\omega Y} & k_{j} \omega \mathrm{e}^{-\omega Y}
\end{array}\right] \\
& \times\left[\prod_{k=j}^{n-1}\left(\frac{1}{r_{k / k+1}} \boldsymbol{G}_{\mathbf{k} / \mathbf{k}+\mathbf{1}}\right)\right]\left[\begin{array}{c}
-\mathrm{e}^{-2 \omega H_{n}} \\
1
\end{array}\right] \cdot \sum_{l=0}^{\infty} p^{l} .
\end{aligned}
$$

Since the solutions in the transformed domain expressed in Eq. (23) are mainly exponential functions of $\omega$, the inverse Fourier transformation can be performed term by term. By omitting the lengthy algebraic derivation, the explicit solutions for temperature and heat flux are obtained as follows:

$$
\begin{gathered}
T^{(j)}(X, Y)=T_{0} \sum_{l=0}^{\infty} \sum_{k=1}^{N} M_{k}^{1}\left(\frac{Y+F_{k}^{a}}{X^{2}+\left(Y+F_{k}^{a}\right)^{2}}\right. \\
\left.+\frac{Y+F_{k}^{b}}{X^{2}+\left(Y+F_{k}^{b}\right)^{2}}\right),
\end{gathered}
$$

$$
\begin{aligned}
q_{Y}^{(j)}(X, Y)=- & T_{0} k_{j} \sum_{l=0}^{\infty} \sum_{k=1}^{N} M_{k}^{1}\left(\frac{X^{2}-\left(Y+F_{k}^{a}\right)^{2}}{\left(X^{2}+\left(Y+F_{k}^{a}\right)^{2}\right)^{2}}\right. \\
& \left.+\frac{X^{2}-\left(Y+F_{k}^{b}\right)^{2}}{\left(X^{2}+\left(Y+F_{k}^{b}\right)^{2}\right)^{2}}\right)
\end{aligned}
$$

$$
\begin{gathered}
q_{X}^{(j)}(X, Y)=2 T_{0} k_{j} \sum_{l=0}^{\infty} \sum_{k=1}^{N} M_{k}^{1}\left(\frac{X\left(Y+F_{k}^{a}\right)}{\left(X^{2}+\left(Y+F_{k}^{a}\right)^{2}\right)^{2}}\right. \\
\left.+\frac{X\left(Y+F_{k}^{b}\right)}{\left(X^{2}+\left(Y+F_{k}^{b}\right)^{2}\right)^{2}}\right)
\end{gathered}
$$

where $N=\left(2^{n-j}\right)\left(2^{n}-1\right)^{l}$. Here $n$ is the number of layers, and $j$ is the $j$ th layer where the solution is required. The terms $M_{k}^{1}, F_{k}^{a}$ and $F_{k}^{b}$ in Eq. (24)-(26) are defined as: 


$$
\begin{aligned}
& \left\{\begin{array}{l}
a_{1}=1, f_{1}^{A_{1}}=-2 H_{n}, f_{1}^{A_{2}}=0, \\
a_{i+2^{k-1}}=a_{i} t_{n-k / n-k+1}, \\
f_{i+2^{k-1}}^{A_{1}}=-\left(f_{i}^{A_{1}}+2 H_{n}+2 H_{n-k}\right), \\
f_{i+2^{k-1}}^{A_{2}}=-\left(f_{i}^{A_{2}}+2 H_{n}-2 H_{n-k}\right),
\end{array}\right. \\
& \quad k=1,2, \ldots n-1, \quad i=1,2, \ldots 2^{k-1}, \\
& \left\{\begin{array}{l}
r_{i}^{p}=-a_{i}, \quad r_{2^{n-1}+i}^{p}=a_{i}, \\
f_{i}^{p}=f_{i}^{A_{2}}, \quad f_{2^{n-1}+i}^{p}=f_{i}^{A_{1}},
\end{array}\right. \\
& \quad \begin{array}{l}
i=1,2, \ldots 2^{n-1}, \\
r_{k}^{l}=\prod_{o=1}^{l} r_{i_{o}}^{p},
\end{array} \\
& i_{k}^{l}=\sum_{o=1}^{l} f_{i_{o}}^{p}, \\
& k=i_{3}, \ldots i_{l}=1,2,3, \ldots 2^{n}-1, \\
& k=\sum_{o=1}^{l-1}\left(i_{o}-1\right)\left(2^{n}-1\right)^{l-o}+i_{l},
\end{aligned}
$$

for $l=0, r_{i_{0}}^{0}=1$ and $g_{i_{0}}^{0}=0$

$$
\begin{aligned}
& \left\{\begin{array}{l}
M_{(i-1)\left(2^{n}-1\right)^{l}+k}^{1}=\frac{1}{2 \pi}\left(\prod_{o=1}^{j-1} r_{o / o+1}\right) \sum_{i=1}^{2^{n-j}\left(2^{n}-1\right)^{l}} \sum_{k=1}^{l} a_{i} r_{k}^{l}, \\
F_{(i-1)\left(2^{n}-1\right)^{l}+k}^{a}=g_{k}^{l}+f_{i}^{A_{1}}, \\
F_{(i-1)\left(2^{n}-1\right)^{l}+k}^{b}=g_{k}^{l}+f_{i}^{A_{2}},
\end{array}\right. \\
& \quad k=1,2, \ldots\left(2^{n}-1\right)^{l}, \quad i=1,2, \ldots 2^{n-j} .
\end{aligned}
$$

Finally, by substituting $X$ and $Y$ defined in Eq. (9) into Eqs. (24)-(26) and using Eq. (5), the explicit expressions of temperature and heat flux fields for anisotropic multi-layered media subjected to a prescribed concentrated temperature $T_{0}$ on the top surface are presented as follows:

$$
\begin{aligned}
T^{(j)}(x, y)= & T_{0} \sum_{l=0}^{\infty} \sum_{k=1}^{N} M_{k}^{1}\left(\frac{Y+F_{k}^{a}}{X^{2}+\left(Y+F_{k}^{a}\right)^{2}}\right. \\
& \left.+\frac{Y+F_{k}^{b}}{X^{2}+\left(Y+F_{k}^{b}\right)^{2}}\right) \\
q_{y}^{(j)}(x, y)=- & T_{0} k_{j} \sum_{l=0}^{\infty} \sum_{k=1}^{N} M_{k}^{1}\left(\frac{X^{2}-\left(Y+F_{k}^{a}\right)^{2}}{\left(X^{2}+\left(Y+F_{k}^{a}\right)^{2}\right)^{2}}\right. \\
& \left.+\frac{X^{2}-\left(Y+F_{k}^{b}\right)^{2}}{\left(X^{2}+\left(Y+F_{k}^{b}\right)^{2}\right)^{2}}\right),
\end{aligned}
$$

$$
\begin{aligned}
q_{x}^{(j)}(x, y) & T_{0} k_{j} \sum_{l=0}^{\infty} \sum_{k=1}^{N} M_{k}^{1}\left(\frac{X\left[\beta_{j}\left(Y+F_{k}^{a}\right)-\alpha_{j} X\right]+\left(Y+F_{k}^{a}\right)\left[\beta X-\alpha_{j}\left(Y+F_{k}^{a}\right)\right]}{\left(X^{2}+\left(Y+F_{k}^{a}\right)^{2}\right)^{2}}\right. \\
& \left.+\frac{X\left[\beta_{j}\left(Y+F_{k}^{b}\right)-\alpha_{j} X\right]+\left(Y+F_{k}^{b}\right)\left[\beta X-\alpha_{j}\left(Y+F_{k}^{b}\right)\right]}{\left(X^{2}+\left(Y+F_{k}^{b}\right)^{2}\right)^{2}}\right)
\end{aligned}
$$

$$
\left[\begin{array}{c}
X \\
Y
\end{array}\right]=\left[\begin{array}{ll}
1 & \alpha_{j} \\
0 & \beta_{j}
\end{array}\right]\left[\begin{array}{l}
x \\
y
\end{array}\right]+\sum_{k=1}^{j-1} h_{k}\left[\begin{array}{c}
\alpha_{k}-\alpha_{k+1} \\
\beta_{k}-\beta_{k+1}
\end{array}\right] .
$$

It is interesting to note that $F_{k}^{a}$ and $F_{k}^{b}$ are dependent on the thickness of the layer, i.e., $H_{j}$, and $M_{k}^{1}$ depends only on the refraction and reflection coefficients, i.e., $r_{j / j+1}$ and $t_{j / j+1}$.

If the concentrated temperature is applied on the bottom surface of the anisotropic multi-layered medium, the boundary conditions become

$$
\left.T^{(1)}\right|_{y=0}=0,\left.\quad T^{(n)}\right|_{y=h_{n}}=T_{0} \delta(x) .
$$

By using the similar method indicated previously, the solutions in the Fourier transformed domain are obtained as follows:

$$
\begin{aligned}
{\left[\begin{array}{c}
\widetilde{T}^{(j)} \\
\tilde{q}_{Y}^{(j)}
\end{array}\right]=} & \frac{\mathrm{e}^{-\mathrm{i} \omega D-\omega H_{n}}}{B_{1}+B_{2} \mathrm{e}^{-2 \omega H_{n}}}\left[\begin{array}{cc}
\mathrm{e}^{\omega Y} & \mathrm{e}^{-\omega Y} \\
-k_{j} \omega \mathrm{e}^{\omega Y} & k_{j} \omega \mathrm{e}^{-\omega Y}
\end{array}\right] \\
& \times\left[\prod_{k=1}^{j-1}\left(\frac{1}{r_{k+1 / k}} G_{n-k+1 / n-k}\right)\right]\left[\begin{array}{c}
1 \\
-1
\end{array}\right]
\end{aligned}
$$

where

$$
\begin{aligned}
& {\left[\begin{array}{l}
B_{1} \\
B_{2}
\end{array}\right]=\left[\prod_{k=1}^{n-1}\left(\frac{1}{r_{k+1 / k}} G_{n-k+1 / n-k}\right)\right]\left[\begin{array}{c}
1 \\
-1
\end{array}\right],} \\
& D=\alpha_{n} h_{n}+\sum_{k=1}^{n-1}\left(\alpha_{k}-\alpha_{k+1}\right) h_{k}, \\
& H_{n}=\beta_{n} h_{n}+\sum_{k=1}^{n-1}\left(\beta_{k}-\beta_{k+1}\right) h_{k} .
\end{aligned}
$$

Note that $D$ is a shifted amount in the horizontal direction of the concentrated temperature and $H_{n}$ is the total thickness of the multi-layered medium after applying the linear coordinate transformation as indicated in Eq. (9).

By using the series expansion technique and the inverse Fourier transformation, the explicit solutions can be expressed as follows:

$$
\begin{aligned}
T^{(j)}(X, Y)= & T_{0} \sum_{l=0}^{\infty} \sum_{k=1}^{N} M_{k}^{2}\left(\frac{Y+F_{k}^{c}}{(X-D)^{2}+\left(Y+F_{k}^{c}\right)^{2}}\right. \\
& \left.+\frac{Y+F_{k}^{d}}{(X-D)^{2}+\left(Y+F_{k}^{d}\right)^{2}}\right) \\
q_{Y}^{(j)}(X, Y)=- & T_{0} k_{j} \sum_{l=0}^{\infty} \sum_{k=1}^{N} M_{k}^{2}\left(\frac{(X-D)^{2}-\left(Y+F_{k}^{c}\right)^{2}}{\left((X-D)^{2}+\left(Y+F_{k}^{c}\right)^{2}\right)^{2}}\right. \\
& \left.+\frac{(X-D)^{2}-\left(Y+F_{k}^{d}\right)^{2}}{\left((X-D)^{2}+\left(Y+F_{k}^{d}\right)^{2}\right)^{2}}\right)
\end{aligned}
$$




$$
\begin{aligned}
q_{X}^{(j)}(X, Y)=2 T_{0} k_{j} \sum_{l=0}^{\infty} \sum_{k=1}^{N} M_{k}^{2}\left(\frac{(X-D)\left(Y+F_{k}^{c}\right)}{\left((X-D)^{2}+\left(Y+F_{k}^{c}\right)^{2}\right)^{2}}\right. \\
\left.+\frac{(X-D)\left(Y+F_{k}^{d}\right)}{\left((X-D)^{2}+\left(Y+F_{k}^{d}\right)^{2}\right)^{2}}\right)
\end{aligned}
$$

where

$$
\begin{aligned}
& \left\{\begin{array}{l}
b_{1}=1 f_{1}^{B}=0, \\
b_{i+2^{k-1}}=b_{i} t_{k+1 / k}, \\
f_{i+2^{k-1}}^{B}=-\left(f_{i}^{B}+2 H_{k}\right),
\end{array}\right. \\
& k=1,2, \ldots m-1, \quad i=1,2, \ldots 2^{k-1}, \\
& \left\{r_{i}^{p}=-b_{i}, \quad r_{2^{n-1}+i}^{p}=b_{i},\right. \\
& \left\{f_{i}^{p}=f_{i}^{B}, \quad f_{2^{n-1}+i}^{p^{n-1}+i}=-f_{i}^{B}-2 H_{n},\right. \\
& i=1,2, \ldots 2^{n-1} \text {, } \\
& \left\{\begin{array}{l}
r_{k}^{l}=\prod_{o=1}^{l} r_{i_{o}}^{p}, \\
g_{k}^{l}=\sum_{o=1}^{l} f_{i_{o}}^{p},
\end{array}\right. \\
& i_{1}, i_{2}, i_{3}, \ldots i_{l}=1,2, \ldots 2^{n}-1, \\
& k=\sum_{o=1}^{l-1}\left(i_{o}-1\right)\left(2^{n}-1\right)^{l-o}+i_{l},
\end{aligned}
$$

for $l=0, r_{i_{0}}^{0}=1$ and $g_{i_{0}}^{0}=0$,

$$
\begin{aligned}
& \left\{\begin{array}{l}
M_{(i-1)\left(2^{n}-1\right)^{l}+k}^{2}=\frac{1}{2 \pi}\left(\prod_{o=1}^{n-1} r_{o+1 / o}\right) \sum_{i=1}^{2^{j-1}} \sum_{k=1}^{\left(2^{n}-1\right)^{l}} b_{i} r_{k}^{l}, \\
F_{(i-1)\left(2^{n}-1\right)^{l}+k}^{c}=g_{k}^{l}+f_{i}^{B}-H_{n}, \\
F_{(i-1)\left(2^{n}-1\right)^{l}+k}^{d}=g_{k}^{l}-f_{i}^{B}-3 H_{n},
\end{array}\right. \\
& \quad k=1,2, \ldots\left(2^{n}-1\right)^{l}, \quad i=1,2, \ldots 2^{j-1},
\end{aligned}
$$

in which,

$t_{j+1 / j}=\frac{k_{j+1}-k_{j}}{k_{j+1}+k_{j}}, \quad r_{j+1 / j}=\frac{2 k_{j+1}}{k_{j}+k_{j+1}}$.

\section{Explicit solutions for distributed temperature on surfaces}

The full-field solutions of anisotropic multi-layered media subjected to concentrated temperature on the surfaces are obtained in the previous section. In this section, the solutions of temperature and heat flux for multi-layered media subjected to distributed temperature on the surfaces will be discussed.
The definition of convolution and the convolution property of Fourier transform are as follows:

$$
\begin{aligned}
& f(x) * g(x)=\int_{-\infty}^{\infty} f(\tau) g(x-\tau) \mathrm{d} \tau, \\
& \mathfrak{\Im}(f(x) * g(x))=\widetilde{F}(\omega) \widetilde{G}(\omega) .
\end{aligned}
$$

By using the convolution property of the Fourier transform and the Green's function in the transformed domain, it is easy to construct solutions for distributed temperature applied in the surfaces. Now consider the case that the top surface on $y=0,|x|<a$ is under the action of a prescribed uniformly distributed temperature, that is, the boundary condition is replaced by

$\left.T^{(1)}\right|_{y=0}=T_{0}\{H(x+a)-H(x-a)\}$,

where $H()$ is the Heaviside function. The boundary condition in the transformed domain is $\widetilde{T}^{(1)}=\frac{2 T_{0} \sin a \omega}{\omega}$. It is easy to write down the complete solution in the Fourier transformed domain as follows:

$$
\begin{aligned}
{\left[\begin{array}{c}
\widetilde{T}^{(j)} \\
\tilde{q}_{Y}^{(j)}
\end{array}\right]=} & \frac{2 T_{0} \sin a \omega}{\omega\left(A_{1}+A_{2}\right)}\left[\begin{array}{cc}
\mathrm{e}^{\omega Y} & \mathrm{e}^{-\omega Y} \\
-k_{j} \omega \mathrm{e}^{\omega Y} & k_{j} \omega \mathrm{e}^{-\omega Y}
\end{array}\right] \\
& \times\left[\prod_{k=j}^{n-1}\left(\frac{1}{r_{k / k+1}} \boldsymbol{G}_{\mathbf{k} / \mathbf{k}+\mathbf{1}}\right)\right]\left[\begin{array}{c}
-\mathrm{e}^{-2 \omega H_{n}} \\
1
\end{array}\right] .
\end{aligned}
$$

The explicit solutions of temperature and heat flux for multi-layered media subjected to a uniformly distributed temperature $T_{0}$ in the region $2 a$ on the top surface are expressed as follows:

$$
\begin{aligned}
T^{(j)}(X, Y)= & T_{0} \sum_{l=0}^{\infty} \sum_{k=1}^{N} M_{k}^{1}\left(\tan ^{-1} \frac{X+a}{Y+F_{k}^{a}}-\tan ^{-1} \frac{X-a}{Y+F_{k}^{a}}\right. \\
& \left.+\tan ^{-1} \frac{X+a}{Y+F_{k}^{b}}-\tan ^{-1} \frac{X-a}{Y+F_{k}^{b}}\right),
\end{aligned}
$$

$q_{Y}^{(j)}(X, Y)=T_{0} k_{j} \sum_{l=0}^{\infty} \sum_{k=1}^{N} M_{k}^{1}$

$$
\times\left(\begin{array}{c}
\frac{X+a}{(X+a)^{2}+\left(Y+F_{k}^{a}\right)^{2}}-\frac{X-a}{(X-a)^{2}+\left(Y+F_{k}^{a}\right)^{2}} \\
+\frac{X+a}{(X+a)^{2}+\left(Y+F_{k}^{b}\right)^{2}}-\frac{X-a}{(X-a)^{2}+\left(Y+F_{k}^{b}\right)^{2}}
\end{array}\right),
$$

$$
\begin{aligned}
q_{X}^{(j)}(X, Y)= & T_{0} k_{j} \sum_{l=0}^{\infty} \sum_{k=1}^{N} M_{k}^{1} \\
& \times\left(\begin{array}{l}
\frac{Y+F_{k}^{a}}{(X+a)^{2}+\left(Y+F_{k}^{a}\right)^{2}}-\frac{Y+F_{k}^{a}}{(X-a)^{2}+\left(Y+F_{k}^{a}\right)^{2}} \\
+\frac{Y+F_{k}^{b}}{(X+a)^{2}+\left(Y+F_{k}^{b}\right)^{2}}-\frac{Y+F_{k}^{b}}{(X-a)^{2}+\left(Y+F_{k}^{b}\right)^{2}}
\end{array}\right),
\end{aligned}
$$

where $M_{k}^{1}, F_{k}^{a}$ and $F_{k}^{b}$ are given in Eqs. (27a)-(27d). 
Similarly, the solutions of anisotropic multi-layered media subjected to a uniformly distributed temperature $T_{0}$ in the region $|x| \leqslant b$ on the bottom surface are obtained as follows:

$$
\begin{aligned}
T^{(j)}(X, Y)= & T_{0} \sum_{l=0}^{\infty} \sum_{k=1}^{N} M_{k}^{2} \\
& \times\left(\begin{array}{l}
\tan ^{-1} \frac{X-D+b}{Y+F_{k}^{c}}-\tan ^{-1} \frac{X-D-b}{Y+F_{k}^{c}} \\
+\tan ^{-1} \frac{X-D+b}{Y+F_{k}^{d}}-\tan ^{-1} \frac{X-D-b}{Y+F_{k}^{d}}
\end{array}\right),
\end{aligned}
$$

$$
\begin{aligned}
q_{Y}^{(j)} & (X, Y) \\
= & T_{0} k_{j} \sum_{l=0}^{\infty} \sum_{k=1}^{N} M_{k}^{2} \\
& \times\left(\begin{array}{l}
\frac{X-D+b}{(X-D+b)^{2}+\left(Y+F_{k}^{c}\right)^{2}}-\frac{X-D-b}{(X-D-b)^{2}+\left(Y+F_{k}^{c}\right)^{2}} \\
+\frac{X-D+b}{(X-D+b)^{2}+\left(Y+F_{k}^{d}\right)^{2}}-\frac{X-D-b}{(X-D-b)^{2}+\left(Y+F_{k}^{d}\right)^{2}}
\end{array}\right),
\end{aligned}
$$

$$
\begin{aligned}
q_{X}^{(j)} & (X, Y) \\
= & T_{0} k_{j} \sum_{l=0}^{\infty} \sum_{k=1}^{N} M_{k}^{2} \\
& \times\left(\begin{array}{l}
\frac{Y+F_{k}^{c}}{(X-D+b)^{2}+\left(Y+F_{k}^{c}\right)^{2}}-\frac{Y+F_{k}^{c}}{(X-D-b)^{2}+\left(Y+F_{k}^{c}\right)^{2}} \\
+\frac{Y+F_{k}^{d}}{(X-D+b)^{2}+\left(Y+F_{k}^{d}\right)^{2}}-\frac{Y+F_{k}^{d}}{(X-D-b)^{2}+\left(Y+F_{k}^{d}\right)^{2}}
\end{array}\right),
\end{aligned}
$$

where $M_{k}^{2}, F_{k}^{c}$ and $F_{k}^{d}$ are given in Eqs. (36a)-(36d).

\section{Numerical results}

By using the analytical explicit solutions developed in the previous sections, numerical calculations of temperature and heat flux are obtained for anisotropic multi-layered media via a computational program. The full-field analysis for the anisotropic layered medium consisting of 10 layers subjected to prescribed temperature on surfaces will be discussed in detail. The thermal conductivities for each layer are listed in Table 1.

Figs. 2-4 show the full-field distributions of temperature and heat fluxes for prescribed uniformly distributed temperature $T_{0}$ on the top surface $-h \leqslant x \leqslant h$, the thickness for each layer is the same and equal to $h$. In the full-field distribution contours, solid lines and dot lines are used to indicate positive and negative values, respectively. In anisotropic multi-layered media, the

\section{Table 1}

The thermal conductivities for the anisotropic ten-layered medium

\begin{tabular}{llcl}
\hline \multirow{2}{*}{ Layer } & \multicolumn{3}{l}{ Thermal conductivity $(\mathrm{W} / \mathrm{m} \mathrm{K})$} \\
\cline { 2 - 4 } & $k_{11}$ & $k_{12}$ & $k_{22}$ \\
\hline 1 & 44.01 & 11.91 & 85.28 \\
2 & 76.56 & 20.63 & 52.73 \\
3 & 30.65 & 3.37 & 28.82 \\
4 & 83.61 & 18.12 & 20.84 \\
5 & 33.67 & 0 & 33.67 \\
6 & 52.73 & 20.63 & 76.56 \\
7 & 28.82 & 3.37 & 30.65 \\
8 & 83.61 & 18.12 & 20.84 \\
9 & 33.67 & 0 & 33.67 \\
10 & 85.28 & 11.91 & 44.01 \\
\hline
\end{tabular}

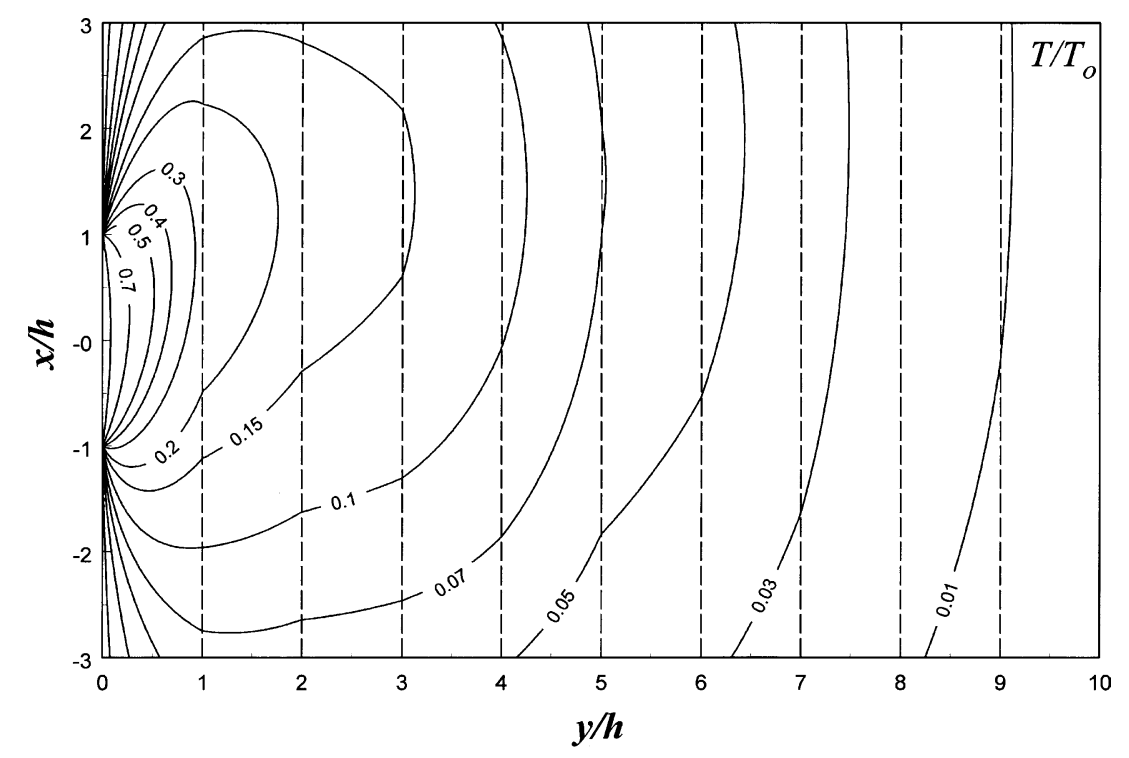

Fig. 2. Full-field temperature distribution for prescribed uniformly distributed temperature $T_{0}$ on the top surface $-h \leqslant x \leqslant h$. 


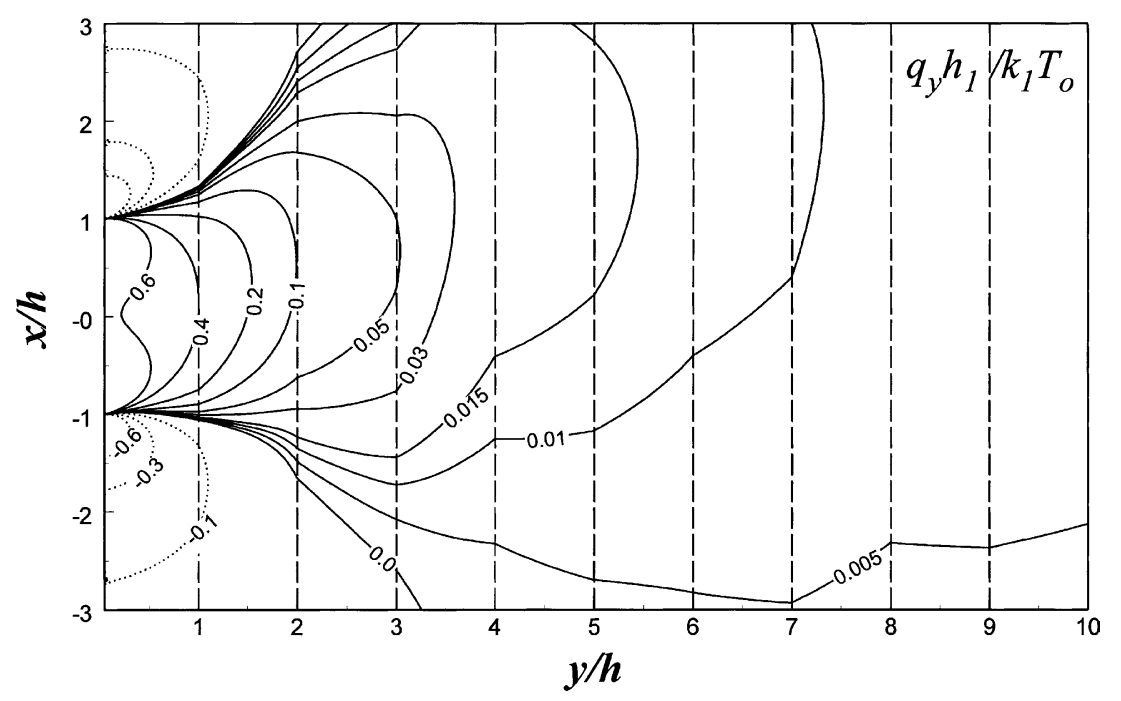

Fig. 3. Full-field heat flux $q_{y}$ distribution for prescribed uniformly distributed temperature $T_{0}$ on the top surface $-h \leqslant x \leqslant h$.

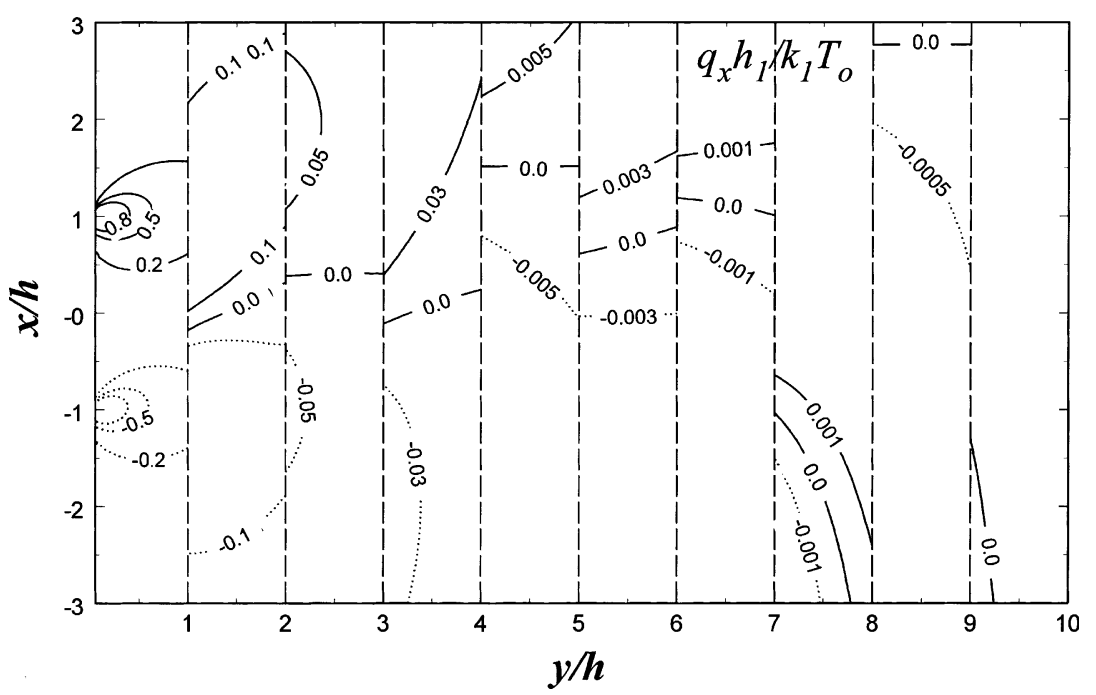

Fig. 4. Full-field heat flux $q_{x}$ distribution for prescribed uniformly distributed temperature $T_{0}$ on the top surface $-h \leqslant x \leqslant h$.

symmetry for the temperature and heat flux fields that is found in the isotropic material is distorted due to the material anisotropy. It is shown in the figures that the temperature and heat flux $q_{y}$ are continuous at the interfaces. This also indicates that the convergence and accuracy for the numerical calculation are satisfied. However, the heat flux $q_{x}$ is discontinuous at the interfaces and the values are small except at the first layer.

Next, the full-field analysis of anisotropic multi-layered media with different layer thickness for each layer is considered. The full-field distributions of temperature and heat flux in the $y$-direction for prescribed uniformly distributed temperature $2 T_{0}$ at two regions $(-2 h \leqslant x \leqslant$ $-h ; h \leqslant x \leqslant 2 h)$ on the top surface and constant temperature $T_{0}$ on the entire bottom surface are shown in Figs. 5 and 6, respectively. Fig. 7 shows the temperature field for prescribed constant temperature $2 T_{0}$ at $-h \leqslant x \leqslant h$ on the top surface and constant temperature $T_{0}$ at $-2 h \leqslant x \leqslant 2 h$ on the bottom surface.

The use of composite materials in a wide variety of modern engineering applications has been rapidly increasing over the past few decades. The increasing use of composite materials in the automotive and aerospace industries has motivated research into solution methods to investigate the thermal properties of these materials. Numerical calculations for layered composites of 12 


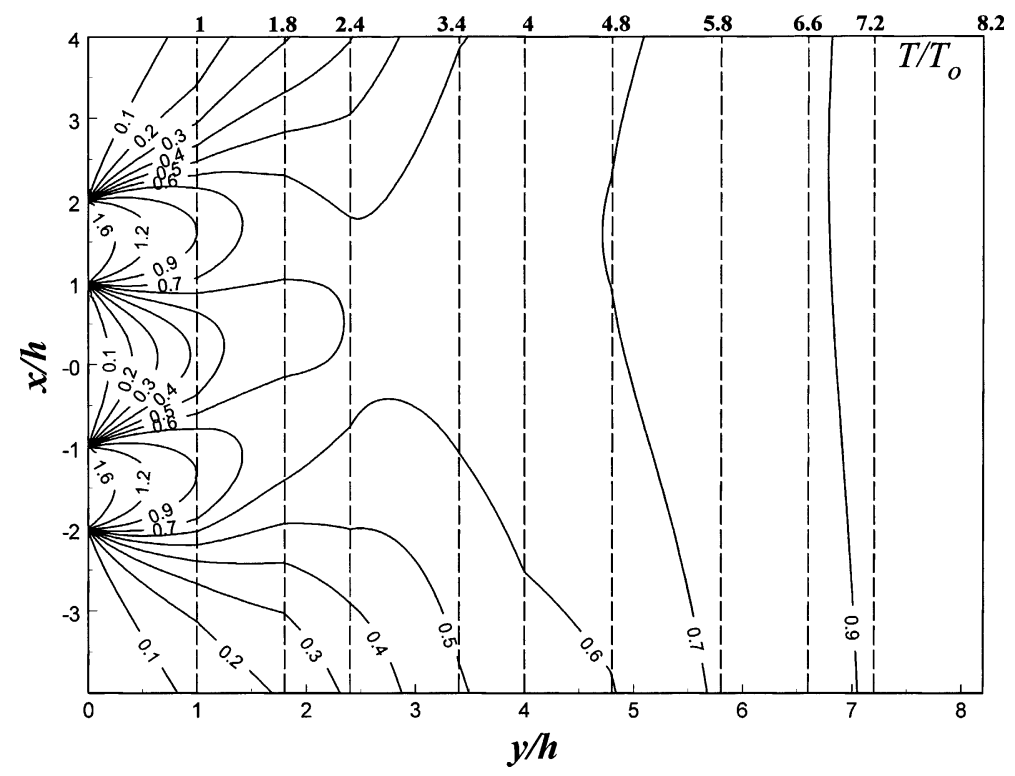

Fig. 5. Full-field temperature distribution for prescribed uniformly distributed temperature $2 T_{0}$ at two regions $(-2 h \leqslant x \leqslant-h$; $h \leqslant x \leqslant 2 h)$ on the top surface and constant temperature $T_{0}$ on the bottom surface.

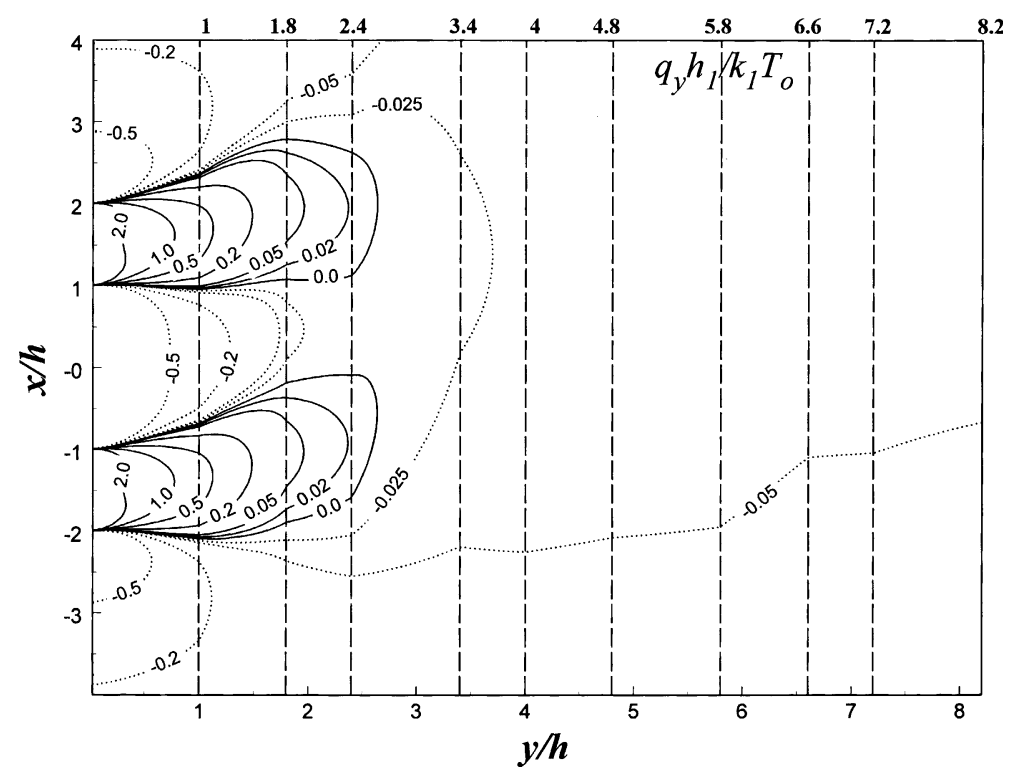

Fig. 6. Full-field heat flux $q_{y}$ distribution for prescribed uniformly distributed temperature $2 T_{0}$ at two regions $(-2 h \leqslant x \leqslant-h$; $h \leqslant x \leqslant 2 h)$ on the top surface and constant temperature $T_{0}$ on the bottom surface.

fiber-reinforced layers will be considered. The fiber angle, $\theta$, is measured counterclockwise from the positive $x$-axis to the fiber direction. A $\left[0^{\circ} / 30^{\circ} / 60^{\circ} / 90^{\circ} / 120^{\circ} / 150^{\circ}\right]_{2}$ laminated composite is considered first. By regarding each layer as being homogeneous and anistotropic, the gross thermal conductivities $k_{11}, k_{12}, k_{22}=30.65,3.37,28.82$
$\mathrm{W} / \mathrm{m} \mathrm{K}$ in the material coordinates of the layer are used. The gross thermal conductivities in the structured coordinates for a given fiber orientation $\theta$ of the layer can be determined via the tensor transformation equation. The numerical result of the temperature distribution for prescribed temperature $2 T_{0}$ in a region $-2 h \leqslant x \leqslant 2 h$ on the 


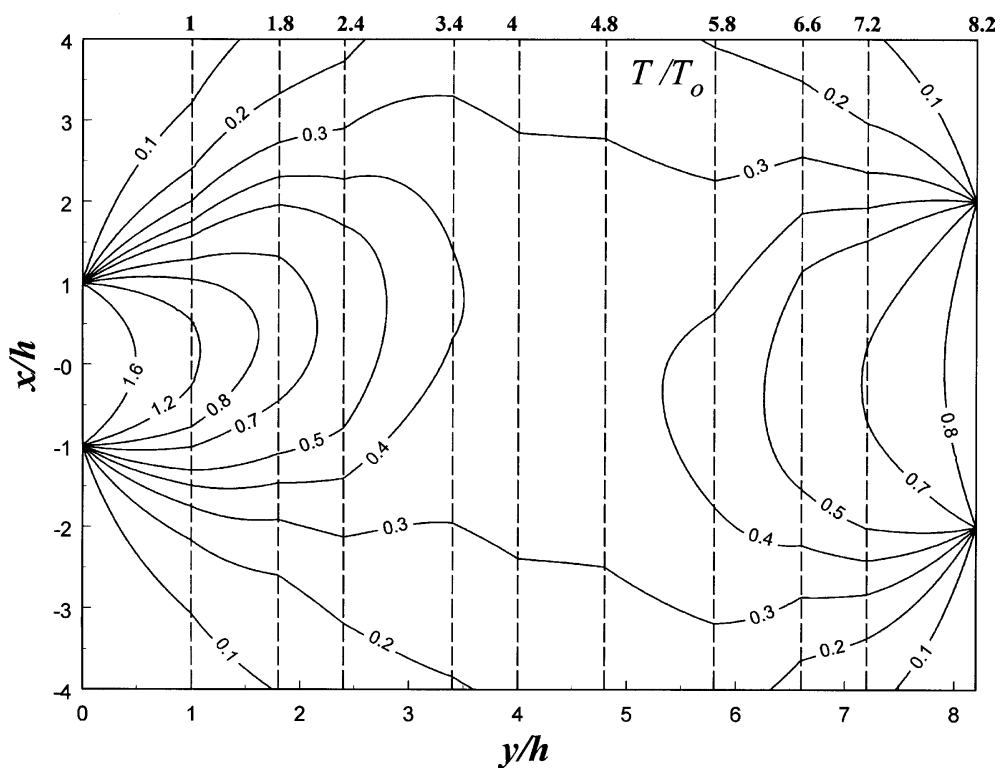

Fig. 7. Full-field temperature distribution for prescribed uniformly distributed temperature $2 T_{0}$ at $-h \leqslant x \leqslant h$ on the top surface and constant temperature $T_{0}$ at $-2 h \leqslant x \leqslant 2 h$ on the bottom surface.

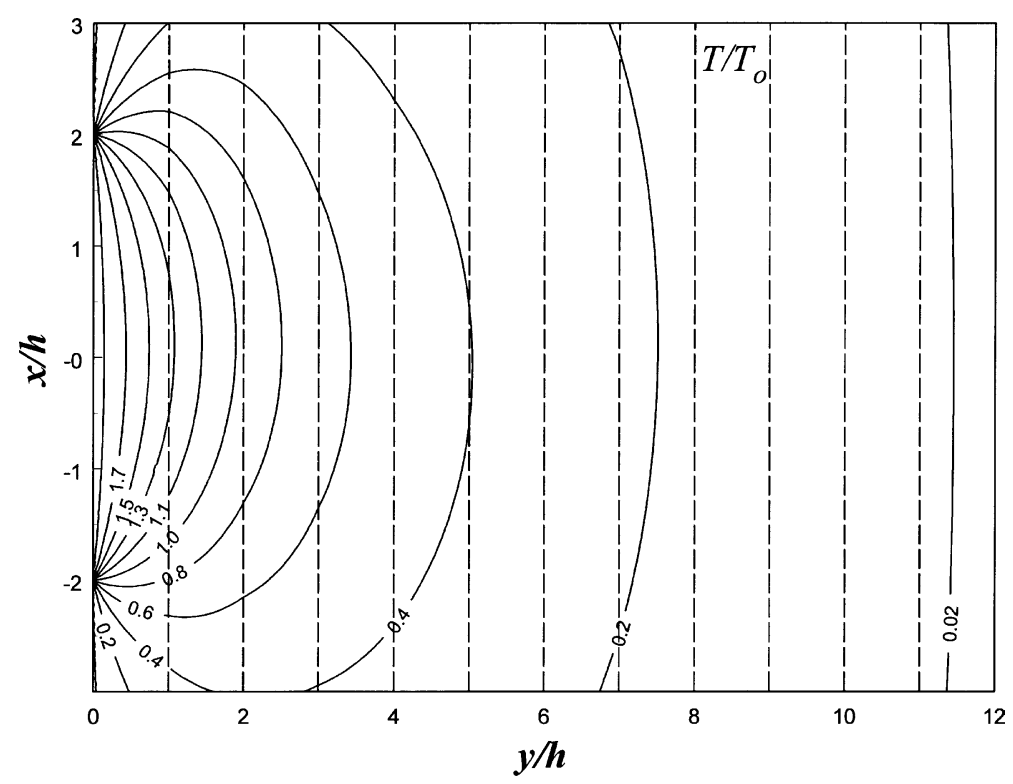

Fig. 8. Full-field temperature distribution of a $\left[30^{\circ} / 60^{\circ} / 90^{\circ} / 120^{\circ} / 150^{\circ}\right]_{2}$ laminated composite for prescribed uniformly distributed temperature $2 T_{0}$ at $-2 h \leqslant x \leqslant 2 h$ on the top surface.

top surface is shown in Fig. 8. Next, a composite layered medium with stacking sequence $\left[0^{\circ} / 60^{\circ} /-60^{\circ}\right]_{2 S}$ is investigated and the result is shown in Fig. 9.

Finally, we consider the prescribed surface temperature as a function in the form

$$
\left.T^{(1)}\right|_{y=0}= \begin{cases}T_{0}\left(1+\cos \frac{\pi}{h} x\right) & |x| \leqslant h \\ 0 & |x|>h .\end{cases}
$$

Figs. 10 and 11 indicate the full-field distributions of temperature and heat flux in the $y$-direction for an 


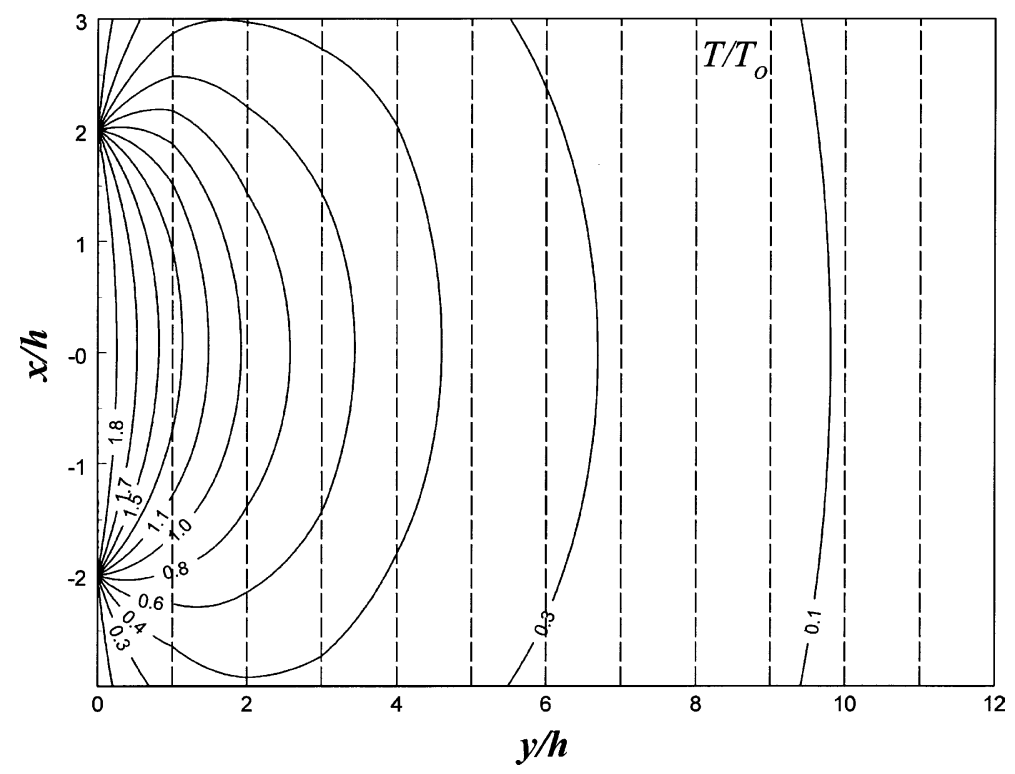

Fig. 9. Full-field temperature distribution of a $\left[0 \% 60 \%-60^{\circ}\right]_{2 S}$ laminated composite for prescribed uniformly distributed temperature $2 T_{0}$ at $-2 h \leqslant x \leqslant 2 h$ on the top surface.

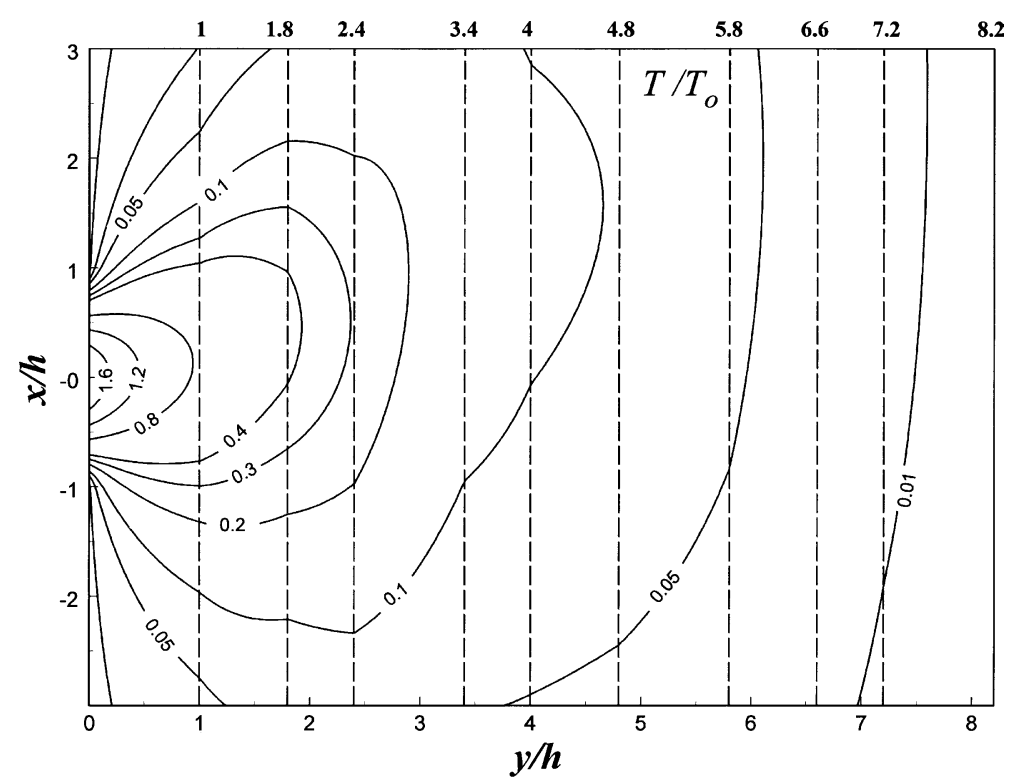

Fig. 10. Full-field temperature distribution for prescribed distributed temperature $T_{0}\left(1+\cos \frac{\pi}{h} x\right)$ at $-h \leqslant x \leqslant h$ on the top surface.

anisotropic layered medium consisting of 10 layers. The thickness for each layer is different and the thermal conductivities are presented in Table 1.

\section{Summary and conclusions}

A two-dimensional steady-state thermal conduction problem of anisotropic multi-layered media is consi- dered in this study. A linear coordinate transformation for multi-layered media is introduced to simplify the governing heat conduction equation without complicating the boundary and interface conditions. The linear coordinate transformation introduced in this study substantially reduces the dependence of the solution on thermal conductivities and the original anisotropic multi-layered heat conduction problem is reduced to an equivalent isotropic problem. By using the Fourier 


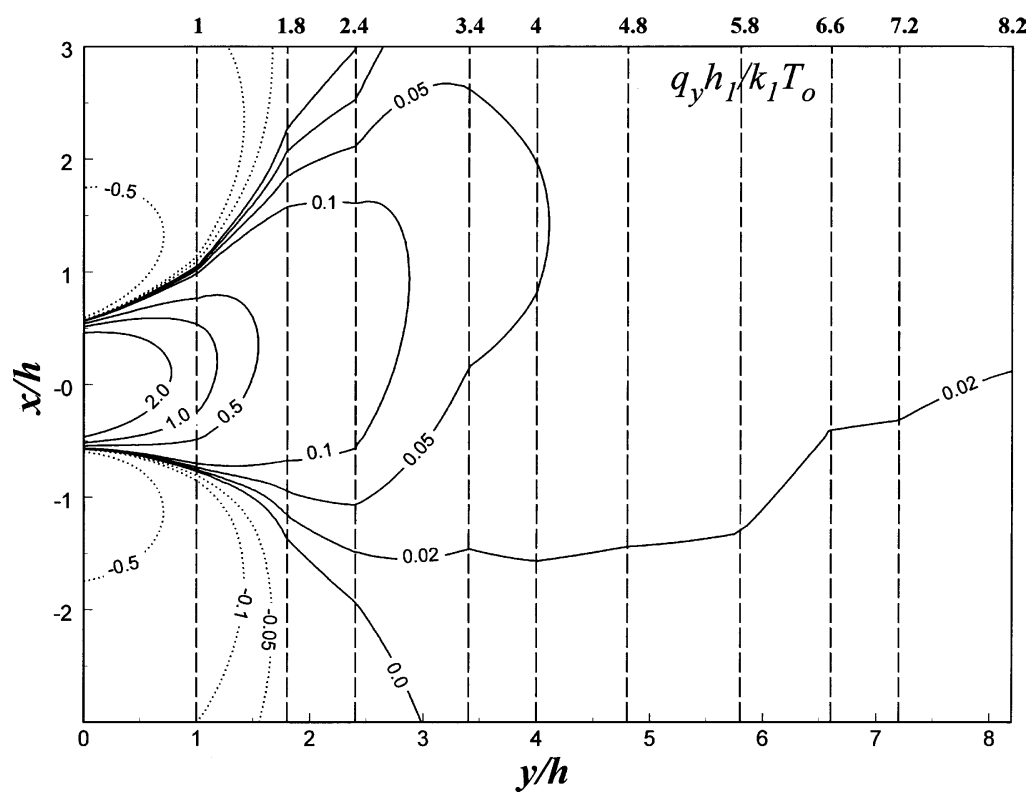

Fig. 11. Full-field heat flux $q_{y}$ distribution for prescribed distributed temperature $T_{0}\left(1+\cos \frac{\pi}{h} x\right)$ at $-h \leqslant x \leqslant h$ on the top surface.

transform technique and a series expansion, exact analytical solutions for the full-field distribution of temperature and heat flux are presented in explicit series forms. The solutions are easy to handle in numerical computation. The numerical results for the full-field distribution for different boundary conditions are presented and are discussed in detail. Solutions for other cases of boundary temperature distribution can be constructed from the basic solution obtained in this study by superposition. The analytical method provided in this study can also be extended to solve the anisotropic heat conduction problem in multi-layered media with embedded heat sources and the results will be given in a follow-up paper.

\section{Acknowledgements}

The financial support of the authors from the National Science Council, People's Republic of China, through grant NSC 89-2212-E002-018 to National Taiwan University is gratefully acknowledged.

\section{References}

[1] H.S. Carslaw, J.C. Jaeger, Conduction of Heat in Solids, Oxford University Press, London, UK, 1959.
[2] M.N. Ozisik, Heat Conduction, Wiley, New York, 1993.

[3] W.A. Wooster, A Textbook in Crystal Physics, Cambridge University Press, London, UK, 1938.

[4] J.F. Nye, Physical Properties of Crystals, Clarendon Press, London, UK, 1957.

[5] T.R. Tauchert, A.Y. Akoz, Stationary temperature and stress fields in an anisotropic elastic slab, J. Appl. Mech. 42 (1975) 647-650.

[6] G.P. Mulholland, B.P. Gupta, Heat transfer in a threedimensional anisotropic solid of arbitrary shape, J. Heat Transfer 99 (1977) 135-137.

[7] Y.P. Chang, Analytical solution for heat conduction in anisotropic media in infinite semi-infinite, and two-placebounded regions, Int. J. Heat Mass Transfer 20 (1977) 1019-1028.

[8] K.C. Poon, Transformation of heat conduction problems in layered composites from anisotropic to orthotropic, Lett. Heat Mass Transfer 6 (1979) 503-511.

[9] K.C. Poon, R.C.H. Tsou, Y.P. Chang, Solution of anisotropic problems of first class by coordinate-transformation, J. Heat Transfer 101 (1979) 340-345.

[10] X.Z. Zhang, Steady-state temperatures in an anisotropic strip, J. Heat Transfer 112 (1990) 16-20.

[11] L. Yan, A.H. Sheikh, J.V. Beck, Thermal characteristics of two-layered bodies with embedded thin-film heat source, J. Electron. Packag. 115 (1993) 276-283.

[12] M.H. Hsieh, C.C. Ma, Analytical investigations for heat conduction problems in anisotropic thin-layer media with embedded heat sources, Int. J. Heat Mass Transfer 45 (2002) 4117-4132. 